\title{
Effect of Astaxanthin supplementation on psychophysiological heart-brain axis dynamics in healthy subjects
}

\author{
Shawn Talbott ${ }^{1}$, Don Hantla ${ }^{2}$, Bob Capelli ${ }^{3}$, Lixin Ding ${ }^{3}$, Yanmei Li $^{4}$, Christian Artaria \\ ${ }^{1}$ EQQIL, Draper, UT, USA; ${ }^{2}$ Treehouse Athletic Club, Draper, UT, USA; ${ }^{3}$ Algae Health Sciences, \\ Irvine, CA, USA; ${ }^{4} \mathrm{BGG}$, Beijing, China; ${ }^{5}$ BGG Europe, Lugano, Switzerland
}

Corresponding author: Shawn Talbott, EQQIL, 648 E Rocky Knoll Draper, UT 84020

Submission Date: July $21^{\text {st }}$, 2019. Acceptance Date: August $28^{\text {th }}$, 2019. Publication Date: August $31^{\text {st }}, 2019$

Citation: Talbott S., Hantla D., Capelli B., Ding L., Li Y., Artaria C. Effect of Astaxanthin supplementation on psychophysiological heart-brain axis dynamics in healthy subjects. Functional Foods in Health and Disease 2019; 9(8): 521-531. DOI: https://doi.org/10.31989/ ffhd.v9i8.636

\section{ABSTRACT:}

Objective: Marine microalgae is the predominant source of natural astaxanthin (NAX), a redorange carotenoid with powerful antioxidant and anti-inflammatory properties. Previous studies suggest that NAX supplementation improves antioxidant capacity and reduces oxidative stress, while also enhancing fat utilization, exercise endurance, cardiovascular function, and neurological parameters. The purpose of this study was to assess the effects of NAX on the psychophysiological "heart-brain-axis" while nutrition (astaxanthin) may impact physiology (cardiovascular function) and psychology (mood state) in a coordinated manner.

Methods: Using a double-blind parallel design, 28 healthy subjects (male=14, female=14, age=42) were supplemented for 8 weeks with NAX (12mg/day Haematococcus pluvialis algal extract) or a matching placebo. Before and after supplementation, subjects performed a cardiovascular stress test (VO2max) and completed a validated Profile of Mood States (POMS) survey to assess global mood state (GM) and related subscales: Vigor (V), Tension (T), Depression (D), Anger (A), Fatigue (F), and Confusion (C).

Results: Subjects in the NAX group showed a significant $\sim 10 \%$ lower average heart rate at submaximal exercise intensities compared to those in the placebo group (aerobic threshold, AeT; NAX 130+17 v. PL 145+14; and anaerobic threshold, AT; NAX 139+20 v. PL 154+11, p<0.05). Significant improvements were found in the NAX group for both positive mood state parameters: GM $(+11 \%, \mathrm{p}<0.05) \& \mathrm{~V}(+5 \%, \mathrm{NS})$; and negative mood state parameters: $\mathrm{T}(-$ $20 \%, \mathrm{NS}), \mathrm{D}(-57 \%, \mathrm{p}<0.05), \mathrm{A}(-12 \%, \mathrm{NS}), \mathrm{F}(-36 \%, \mathrm{p}<0.05)$, and C $(-28 \%, \mathrm{NS})$. 
Conclusions: NAX supplementation lowered average heart rate at submaximal endurance intensities (suggesting a "physical" heart benefit) and improved mood state parameters (suggesting a "mental" brain benefit). While previous studies have shown NAX supplementation to improve parameters associated with heart health (antioxidant, fat oxidation, endurance) and brain health (neuro-inflammation, cognition, antidepressant/anxiolytic), these results suggest that natural astaxanthin supplementation supports the psychophysiological "heart-brain-axis" with simultaneous improvements in both physical and mental wellness.

Keywords: Antioxidant; Carotenoid; Cardiovascular; Mood State; Mental Wellness

\section{INTRODUCTION}

Natural astaxanthin (NAX) is a red-orange carotenoid that provides characteristic color to pink flamingo feathers, pink shrimp shells, and red salmon flesh. The high level of NAX in these tissues is reflective of the animal's dietary intake of NAX from microalgae, copepods, krill, and other lower food chain organisms $[1,2]$. The primary dietary source of astaxanthin for humans is from seafood such as salmon, shrimp, crabs, lobster [3-5]. NAX can exist in 3 stere oisomeric forms [2], two chiral (3S / 3'S and 3R / 3'R), and one meso (3R / 3'S), which are found in nature in variable ratios depending on the diet of the animal (e.g. wild salmon consuming natural algae versus farmed salmon consuming synthetic AX). Synthetic astaxanthin (SAX), derived from petrochemicals and approved as a food coloring for fish aquaculture, is equimolar which results in farmed salmon having high levels of synthetic 3R/3'S, while wild salmon have predominantly $3 \mathrm{~S} / 3$ 'S [6].

NAX can be produced commercially from the microalgae Haematococcus pluvialis (H. pluvialis) and is the only source currently approved for human consumption [6] by both the FDA [US Food and Drug Administration] and EFSA [European Food Safety Authority] (both in dried form or extracted with ethanol or supercritical $\mathrm{CO} 2$ ). $H$. pluvialis as a source of NAX provides the benefits of esterified 3S/3'S astaxanthin stereoisomers (96\% of carotenoids) plus other naturallyoccurring carotenoids (beta-carotene, lutein, zeaxanthin), which may provide synergistic benefits and improved absorption compared to SAX [7, 8]. In extensive pre-clinical trials, NAX has demonstrated far superior antioxidant capacity in vitro and improved health benefits in animals to SAX [9]. In addition, SAX has not been studied for safety directly in humans [9]. Based on its superiority in bioactivity and extensive safety testing in humans, NAX is the form of Astaxanthin that is used in human nutrition by the vast majority of supplement brands and in functional foods and drinks.

NAX has been studied for its antioxidant, anti-inflammatory, cardioprotective, and neuroprotective activities in humans [10-14]. Rodent studies have further shown NAX to reduce blood pressure and improve blood flow [15, 16], possibly via modulation of cellular stress pathways, including nuclear factor $\mathrm{kB}(\mathrm{NFkB})$, nuclear factor E2 related factor 2 (Nrf2), and nitric oxide [17].

In terms of exercise effects, astaxanthin has shown positive results for reducing lactic acid accumulation, increasing fat oxidation/metabolism, and improving endurance performance with most studies demonstrating benefits [18-28]. Many of these effects may be attributed to a 
hypothesized mitochondrial-centric mechanism, which could improve energy and redox cellular metabolism (e.g. via Nrf2-ARE pathway activation). Indeed, mitochondrial redox metabolism has been implicated in various neurological disorders including Alzheimer's, Parkinson's, and agerelated dementia, so it is logical that NAX has been suggested to play a putative prophylactic role in cardiovascular as well as neurologic conditions [29-40].

The heart is recognized to play a vital role not only as a circulatory pump, but as part of a psychophysiological network as a generator and transmitter of system-wide information throughout multiple body systems, including the nervous system [41-46]. Electrical input from the heart can dynamically influence homeostatic, cognitive, perceptual, and emotional processing in the brain, thereby having the potential to affect myriad aspects of mood and behavior [41-44]. Thayer and Lane [41] reviewed the direct and indirect connections between the heart and the brain, including the physiological, behavioral, emotional, and cognitive processes involved in bidirectional control of cortical/cardiac function. Organ crosstalk between the brain/heart has been noted in stress-related cardiomyopathy syndromes and traumatic brain injury [42]. In addition, the use of heart rate variability (HRV) has been recognized for its importance in gauging both the state of the heart (physical stress) as well as the state of the brain (psychological stress). Yoga, as an intervention to restore balanced heart-brain crosstalk through plasticity and stability of the autonomic nervous system, has been used to reduce anxiety levels, atrial fibrillation episodes, blood pressure and neurocardiogenic syncope [43]. Studies have also shown that positive emotional states may improve function of both the cardiovascular and immune systems [45, 46].

\section{MATERIAL AND METHODS}

\section{Participants}

Thirty healthy, active subjects were recruited and split evenly between males and females. Two subjects, one from each group, withdrew from the study due to issues unrelated to the supplement or trial, resulting in 28 subjects that completed the 8-week supplementation period (age $42 \pm 8$, range $26-63$ years; height $169 \pm 10 \mathrm{~cm}$; BW 69 $\pm 6 \mathrm{~kg}$ ). All participants completed informed consent documents approved by an external ethics review board.

\section{Dietary Supplement}

Participants were randomly divided in double-blind fashion into two groups to receive either the natural astaxanthin (NAX) supplement (AstaZine® Natural Astaxanthin, BGG / AlgaeHealth Sciences) or matching placebo (PL). The NAX supplement provided $12 \mathrm{mg} /$ day of natural astaxanthin extracted with ethanol from Haematococcus pluvialis suspended in edible MCT oil (medium chain triglyceride) with d-alpha tocopherol as an antioxidant. Subjects consumed NAX or PL daily for 8 weeks. No adverse events related to the dietary supplement were reported.

\section{Heart/Brain Axis Assessment}

Before and after the supplementation period, subjects performed a VO2max assessment on a treadmill to measure cardiovascular performance (CardioCoach, Korr Medical Technologies). Subjects also completed a psychological mood state survey (POMS, Profile of Mood States) to assess Global Mood State and 6 related subscales: Vigor (V), Tension (T), Depression (D), Anger (A), Fatigue (F), and Confusion (C). The VO2max assessment was designed for participants to reach maximal oxygen consumption and voluntary fatigue within 15 minutes. The protocol 
consisted of a gradual warmup of easy walking/jogging, followed by progressive increases in speed and incline each minute until exhaustion. Heart rate (beats per minute, bpm) and oxygen consumption $(\mathrm{ml} / \mathrm{kg} / \mathrm{min}$ ) were recorded at maximum and two submaximal levels (aerobic threshold, AeT and anaerobic threshold, AT). The POMS survey is a self-administered 64-question assessment that takes approximately 20 minutes to complete and was collected before and after the 8-week supplementation period.

\section{Data Management and Analysis}

All participant data was maintained in a central location and transcribed to a central database. The data was identified by subject number and examined for accuracy and completeness. Tabulated data was analyzed with JMP 8.0 (SAS Institute, Cary, NC) using standard parametric paired t tests, and significance was assessed with a 2-tailed alpha level set at 0.05 .

\section{RESULTS}

Subject baseline characteristics are presented in Table 1. There was no improvement in maximal oxygen uptake (VO2max while running) with NAX supplementation (Figure 1). However, subjects in the NAX group showed a significant $\sim 10 \%$ lower average heart rate at submaximal intensities (aerobic threshold and anaerobic threshold) compared to both pre-supplementation values and compared to the placebo group (Figure 2), suggesting a profound "cardiotonic" effect of NAX supplementation with superior metabolic efficiency at submaximal aerobic endurance intensities. Aerobic threshold (AeT) and anaerobic threshold (AT) heart rates were significantly lower post-supplementation of $\quad$ NAX versus PL (AeT; NAX 130 \pm 17 v. PL 145 \pm 14 ; and AT; NAX 139 \pm 20 v. PL $154 \pm 11, p<0.05$ ), indicating that NAX subjects were able to perform a certain level of physical work at a lower relative intensity or higher cardiovascular efficiency.

Table 1. Baseline subject characteristics Data represent average (Mean) values $( \pm \mathrm{SD})$. NAX, natural astaxanthin group; PL, placebo group; VO2max, maximal

\begin{tabular}{|l|l|l|}
\hline & NAX & PL \\
\hline Height $(\mathrm{cm})$ & $169(11)$ & $168(9)$ \\
\hline Weight $(\mathrm{kg})$ & $70.0(7.1)$ & $69.3(7.0)$ \\
\hline Body Fat $(\%)$ & $20.3(6.3)$ & $24.9(8.4)$ \\
\hline VO2max $(\mathrm{ml} / \mathrm{kg} / \mathrm{min})$ & $43.7(9.1)$ & $42.9(7.2)$ \\
\hline Peak HR $(\mathrm{bpm})$ & $169(9)$ & $172(9)$ \\
\hline AeT VO2 $(\mathrm{ml} / \mathrm{kg} / \mathrm{min})$ & $33.5(7.5)$ & $31.3(3.8)$ \\
\hline AeT HR $(\mathrm{bpm})$ & $145(13)$ & $143(11)$ \\
\hline AT VO2 $(\mathrm{ml} / \mathrm{kg} / \mathrm{min})$ & $38.5(8.3)$ & $36.2(4.7)$ \\
\hline AT HR $(\mathrm{bpm})$ & $154(15)$ & $153(11)$ \\
\hline
\end{tabular}

NAX, natural astaxanthin group; PL, placebo group; VO2max, maximal oxygen consumption; Peak HR, heart rate at VO2max; AeT, aerobic threshold; AT, anaerobic threshold. 
There was no improvement in maximal oxygen uptake (VO2max while running) with NAX supplementation (Figure 1).

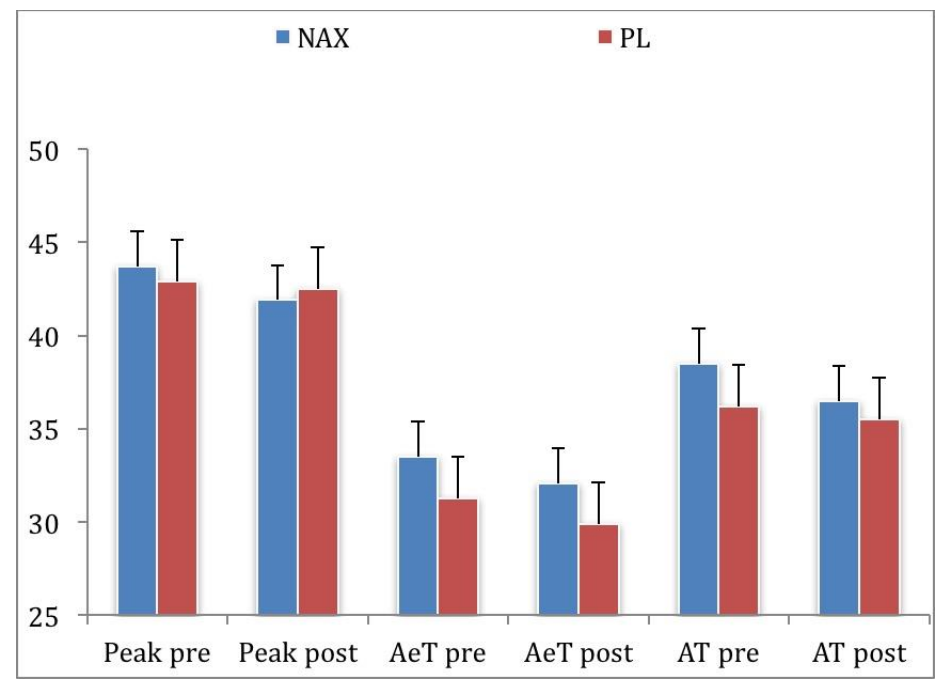

Figure 1. Oxygen consumption at baseline (pre-supplementation) and week 8 (postsupplementation. There were no significant differences in absolute oxygen consumption at any time point. NAX, natural astaxanthin group; PL, placebo group; pre, baseline; post, week 8; Peak, maximal oxygen consumption; AeT, aerobic threshold; AT, anaerobic threshold.

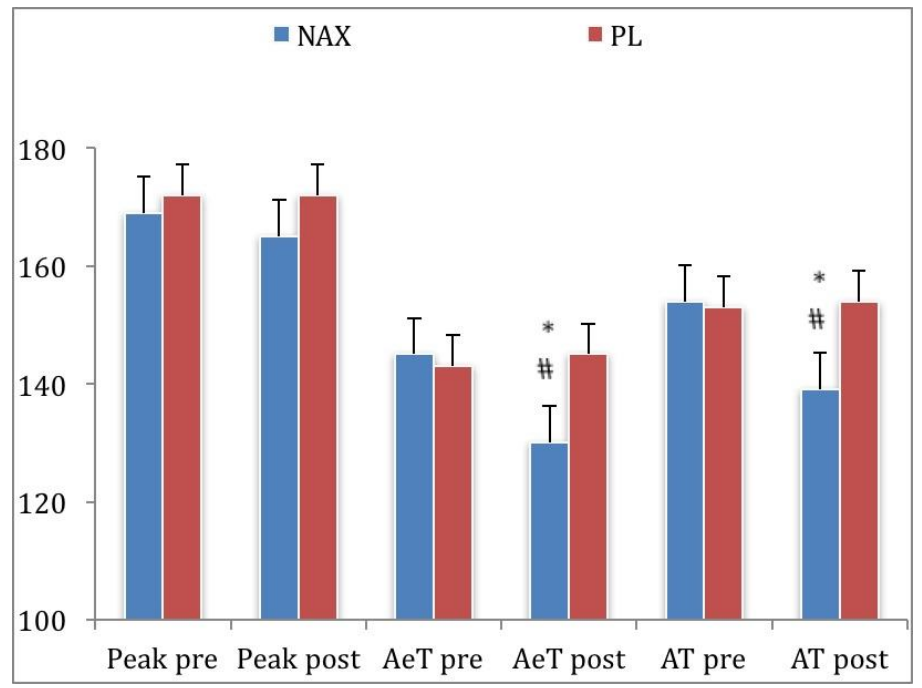

Figure 2. Heart Rate at baseline (pre-supplementation) and week 8 (post-supplementation). At submaximal running intensity (AeT, aerobic threshold and AT, anaerobic threshold), the NAX group had significantly lower heart rate (HR) compared to pre-supplementation values $\left(^{*}\right)$ and compared to placebo $(\mathrm{PL})$ values (\#, both $\mathrm{p}<0.05$ ) after 8 weeks (post) of supplementation with $12 \mathrm{mg} /$ day of natural astaxanthin.

NAX, natural astaxanthin group; PL, placebo group; HR, heart rate; pre, baseline; post, week 8; Peak, maximal HR at VO2max; AeT, aerobic threshold; AT, anaerobic threshold.

However, subjects in the NAX group showed a significant $\sim 10 \%$ lower average heart rate at submaximal intensities (aerobic threshold and anaerobic threshold) compared to both presupplementation values and compared to placebo (Figure 2), suggesting a profound "cardiotonic" effect of NAX supplementation with superior metabolic efficiency at submaximal aerobic 
endurance intensities. Aerobic threshold (AeT) and anaerobic threshold (AT) heart rates were significantly lower post-supplementation in NAX versus PL (AeT; NAX 130 17 v. PL 145 \pm 14 ; and AT; NAX 139 \pm 20 v. PL 154 $\pm 11, \mathrm{p}<0.05)$, indicating that NAX subjects were able to perform a certain level of physical work at a lower relative intensity or higher cardiovascular efficiency.

In addition, significant improvements were found in NAX for some positive mood state parameters (Figure 3): Global Mood $(+11 \%, \mathrm{p}<0.05)$ \& Vigor $(+5 \%$, NS); and some negative mood state parameters: Tension $(-20 \%$, NS), Depression $(-57 \%, \mathrm{p}<0.05)$, Anger $(-12 \%$, NS), Fatigue $(-36 \%, \mathrm{p}<0.05)$, and Confusion $(-28 \%$, NS), indicating a beneficial psychological effect of NAX on overall mood and specifically in reducing depression and fatigue.

Figure 3. Psychological Mood State at baseline (pre-supplementation) and week 8 (postsupplementation).

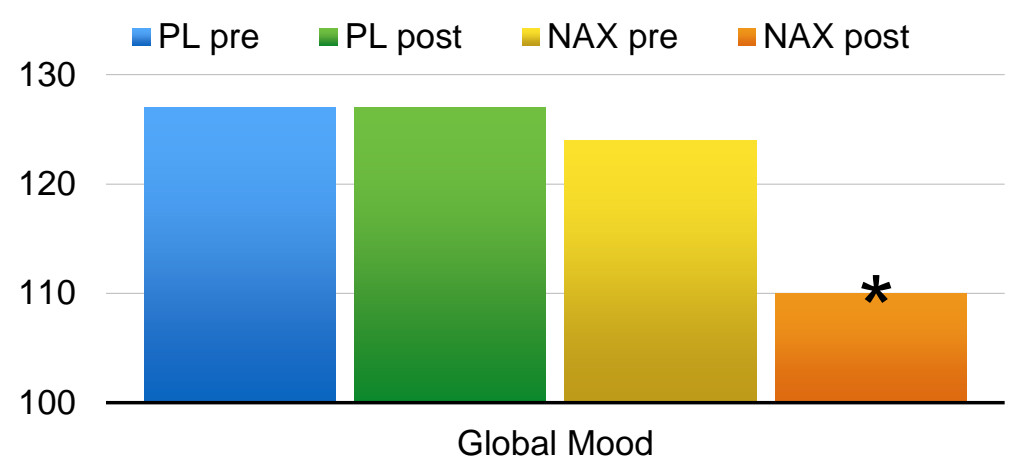

Figure 3a. Improvements were found in NAX for Global Mood $(-11 \%, \mathrm{NAX}=127+20 \mathrm{v}$. $\mathrm{PL}=127+20 ; \mathrm{p}<0.05) * *$ Global Mood State $=$ a lower score indicates a more positive psychological mood state.

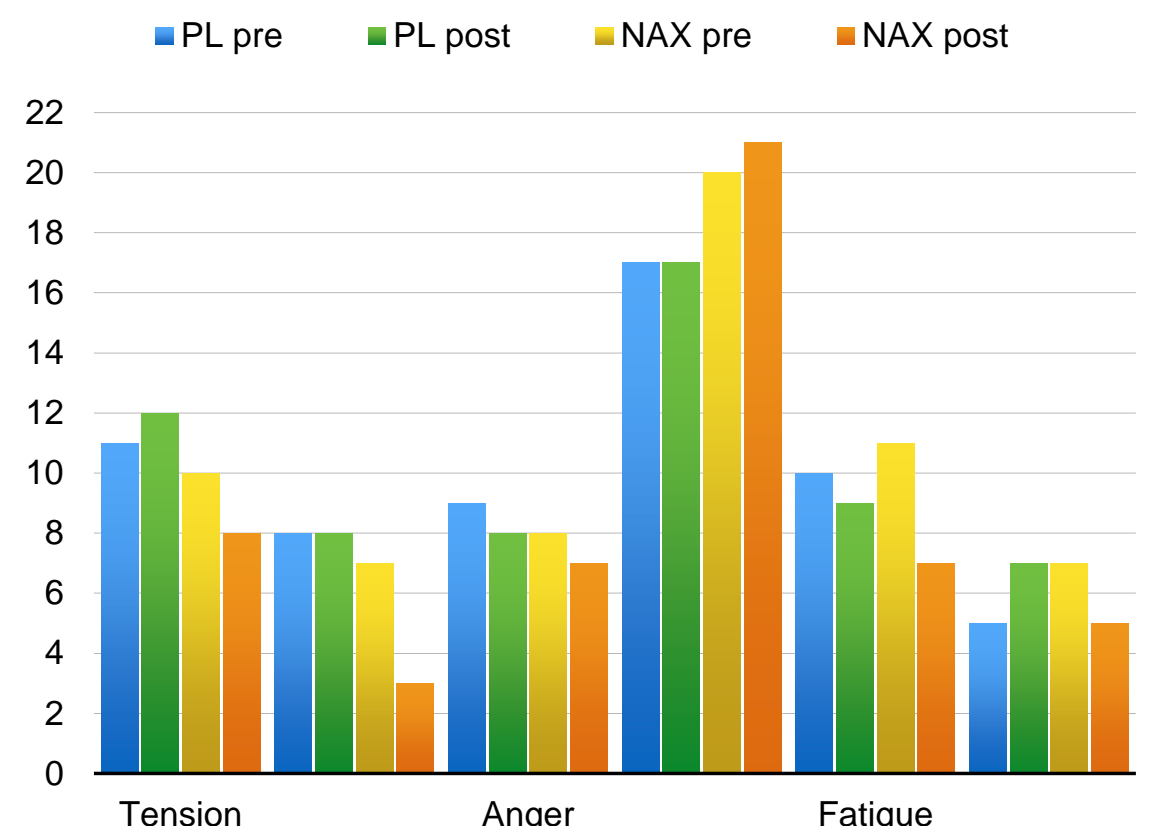

Figure 3b: Improvements were found in NAX for some mood state subscales: Tension (-20\%, NS), Depression (-57\%, p<0.05), Anger (-12\%, NS), Vigor (+5\%, NS), Fatigue $(-36 \%, \mathrm{p}<0.05)$, and Confusion (-28\%, NS). 


\section{DISCUSSION}

Astaxanthin (AX) is a naturally occurring carotenoid, synthesized primarily by marine microalgae, with powerful antioxidant and anti-inflammatory properties. In mammals, dietary AX accumulates in muscle, where it attenuates muscle damage and inhibits peroxidation of DNA and lipids due to prolonged exercise [18-20]. In addition, AX has been identified as a nutrient that may strongly stimulate fat oxidation during exercise. AX supplementation of mice (4-5 weeks with $6-30 \mathrm{mg} / \mathrm{kg}$ BW) improves fat utilization and increases swimming and treadmill running time to exhaustion $[20,21]$. These effects were theorized to be attributable to an improved mitochondrial capacity for fatty acyl-CoA uptake via an improvement in carnitine palmitoyltransferase 1 (CPT1) function, subsequent to inhibition of oxidative damage to the mitochondrial membrane. Such pre-clinical results provide suggestive evidence that AX may have potential ergogenic effects for endurance athletes.

Previous studies of NAX administration in animal models have shown a decrease in exerciseinduced damage to skeletal and cardiac muscle, as well as an increase in redox balance, fat oxidation and time to exhaustion during exercise [18-23]. Some positive rodent studies have administered NAX at fairly high doses of $6-30 \mathrm{mg} / \mathrm{kg}$ [19-22], while others have used lower amounts $(1 \mathrm{mg} / \mathrm{kg})$ to delay physical exhaustion and improve redox balance [24] - relatively higher than the amounts of NAX supplemented in human trials (2-20mg/day).

Humans studies of NAX supplementation have noted improved antioxidant status as well as reduced oxidative damage in sedentary obese subjects $[26,27]$ and untrained men [28]. In athletes, NAX supplementation for 4 weeks reduced lactic acid accumulation following $1200 \mathrm{~m}$ of running [23]. Earnest et al. [25] found significant improvements in power output $(+15 \%=20 \mathrm{~W}$ mean power increase) and faster completion of a $20 \mathrm{~km}$ cycling time trial $(5 \%=2 \mathrm{~min}$ mean change $)$ following NAX supplementation $(4 \mathrm{mg} / \mathrm{day}$ for 4 weeks).

Jiang et al [29] recently demonstrated the anti-depressant effects of NAX, subsequent to serotonergic and anti-inflammatory effects, in an animal model of stress-induced depression. These and other authors suggest that NAX may be advantageous in neuroprotection due to its ability to locate inside the phospholipid membrane and at the membrane surface, as well as its facility in crossing the blood-brain barrier [29-32], which can lead to a wide range of potential psychological benefits including enhanced cognitive function [33, 37, 39], reduced depression [33], lower anxiety [35, 36], and neuroprotection [39, 40].

The current study found intriguing psychological mood state benefits as well as physical cardiotonic benefits of NAX supplementation. At submaximal exercise intensities, supplementation with $12 \mathrm{mg}$ /day of NAX for 8 weeks significantly reduced heart rate at the same relative workload at aerobic threshold (AeT) and anaerobic threshold (AT). Submaximal exercise heart rates were $\sim 10 \%$ lower following NAX supplementation, where average heart rates were 130-145 bpm (aerobic threshold) to 139-154 bpm (anaerobic threshold), but not at higher "peak" intensities (e.g. 165-172 bpm at peak VO2max). Combined with promoting significantly higher overall mood (Global Mood State) and reduced depression and fatigue, NAX supplementation delivered intriguing support of the psychophysiological axis between the brain and the heart.

\section{CONCLUSIONS}

NAX supplementation lowered average heart rate at submaximal endurance intensities (suggesting a "physical" heart benefit) and improved mood state parameters (suggesting a "mental" brain 
benefit). While previous studies have demonstrated how NAX supplementation can improve parameters associated with heart health (antioxidant, fat oxidation, endurance) and brain health (neuro-inflammation, cognition, antidepressant/anxiolytic), these results are the first to show that natural astaxanthin supplementation supports the psychophysiological "heart-brain-axis" with simultaneous improvements in both physical and mental wellness.

In addition to these "psychophysiological" benefits for the heart/brain axis, these results suggest that NAX supplementation may also be a beneficial ergogenic aid for long-distance and ultra-distance endurance athletes (e.g. marathon runners, Ironman triathletes, and ultrarunners/cyclists), where both physiological endurance and psychological balance are simultaneously stressed and the support of both may be a novel approach to improving physical/mental performance.

Because of the dual nature of NAX benefits on heart and brain health, future studies are warranted in expanded populations including elderly subjects and those with cardiac and neurologic complications including post-myocardial infarction, heart failure, statin usage, mitochondrial dysfunction, chronic fatigue, and related conditions.

List of Abbreviations: NAX: natural astaxanthin; POMS: Profile of Mood States; VO2max: maximal oxygen consumption; AeT: aerobic threshold; AT: anaerobic threshold

\section{Acknowledgments}

Funding and astaxanthin supplements for this study were provided by Algae Health Sciences, a division of BGG.

\section{Disclosure}

This work was funded by Algae Health Sciences, a division of BGG. Talbott was principal investigator and reports no conflicts of interest in this work. Hantla reports no conflict of interest. Capelli and Ding are employees of Algae Health Sciences, and Li and Artaria are employees of BGG and BGG Europe respectively.

\section{REFERENCES}

1. Fassett RG, Coombes JS: Astaxanthin in cardiovascular health and disease. Molecules 2012, 17(2):2030-48.

2. Ambati RR, Phang SM, Ravi S, Aswathanarayana RG: Astaxanthin: sources, extraction, stability, biological activities and its commercial applications--a review. Mar Drugs 2014, 12(1):128-52.

3. Guerin M, Huntley ME, Olaizola M: Haematococcus astaxanthin: applications for human health and nutrition. Trends Biotechnol 2003, 21(5):210-216.

4. Higuera-Ciapara I, Felix-Valenzuela L, Goycoolea FM: Astaxanthin: a review of its chemistry and applications. Crit Rev Food Sci Nutr 2006, 46(2):185-196

5. Hussein G, Sankawa U, Goto H, Matsumoto K, Watanabe H: Astaxanthin, a carotenoid with potential in human health and nutrition. J Nat Prod 2006, 69(3):443-449

6. Megdal PA, Craft NA, Handelman GJ: A simplified method to distinguish farmed (Salmo salar) from wild salmon: fatty acid ratios versus astaxanthin chiral isomers. Lipids 2009, 44(6):569-76. 
7. Shah MMR, Liang Y, Cheng JJ, Daroch M: Astaxanthin-Producing Green Microalga Haematococcus pluvialis: From Single Cell to High Value Commercial Products. Front Plant Sci 2016, 7:531.

8. Okada Y, Ishikura M, Maoka T: Bioavailability of astaxanthin in Haematococcus algal extract: the effects of timing of diet and smoking habits. Biosci Biotechnol Biochem 2009, 73(9):1928-32.

9. Capelli B, Talbott S, Ding L: Astaxanthin sources: Suitability for human health and nutrition. Functional Foods in Health and Disease 2019, 9(6):430-445.

10. Rufer CE, Moeseneder J, Briviba K, Rechkemmer G, Bub A: Bioavailability of astaxanthin stereoisomers from wild (Oncorhynchus spp.) and aquacultured (Salmo salar) salmon in healthy men: a randomised, double-blind study. Br J Nutr 2008, 99(5):1048-54.

11. Naguib YM: Antioxidant activities of astaxanthin and related carotenoids. J Agric Food Chem 2000, 48(4):1150-4.

12. Ohgami K, Shiratori K, Kotake S, Nishida T, Mizuki N, Yazawa K, Ohno S: Effects of astaxanthin on lipopolysaccharide-induced inflammation in vitro and in vivo. Invest Ophthalmol Vis Sci 2003, 44(6):2694-701.

13. Pashkow FJ, Watumull DG, Campbell CL: Astaxanthin: a novel potential treatment for oxidative stress and inflammation in cardiovascular disease. Am J Cardiol 2008, 101(10A): 58D-68D.

14. Park JS, Chyun JH, Kim YK, Line LL, Chew BP: Astaxanthin decreased oxidative stress and inflammation and enhanced immune response in humans. Nutr Metab (Lond) 2010, 7:18.

15. Spiller GA, Dewell A: Safety of an astaxanthin-rich Haematococcus pluvialis algal extract: a randomized clinical trial. J Med Food 2003, 6(1):51-6.

16. Hussein G, Goto H, Oda S, Iguchi T, Sankawa U, Matsumoto K, Watanabe H: Antihypertensive potential and mechanism of action of astaxanthin: II. Vascular reactivity and hemorheology in spontaneously hypertensive rats. Biol Pharm Bull 2005, 28(6):967-71.

17. Hussein G, Goto H, Oda S, Sankawa U, Matsumoto K, Watanabe H: Antihypertensive potential and mechanism of action of astaxanthin: III. Antioxidant and histopathological effects in spontaneously hypertensive rats. Biol Pharm Bull 2006, 29(4):684-8.

18. Farruggia C, Yang Y, Kim B, Pham T, Bae M, Park YK, Lee JY: Astaxanthin plays anti-inflammatory and antioxidant effects by inhibiting NFkB nuclear translocation and NOX2 expression in macrophages. FASEB J 2015, 29(Suppl 1):603.8

19. Aoi W, Naito Y, Sakuma K, Kuchide M, Tokuda H, Maoka T, Toyokuni S, et al.: Astaxanthin limits exercise-induced skeletal and cardiac muscle damage in mice. Antioxid Redox Signal 2003, 5:139-144

20. Aoi W, Naito Y, Takanami Y, Ishii T, Kawai Y, Akagiri S, Kato Y, et al.: Astaxanthin improves muscle lipid metabolism in exercise via inhibitory effect of oxidative CPT I modification. Biochem Biophys Res Commun 2008, 366:892-897

21. Ikeuchi M, Koyama T, Takahashi J, Yazawa K: Effects of astaxanthin supplementation on exercise-induced fatigue in mice. Biol Pharm Bull 2006, 29(10):2106-10. 
22. Ikeuchi M, Koyama T, Takahashi J, Yazawa K: Effects of astaxanthin in obese mice fed a high-fat diet. Biosci Biotechnol Biochem 2007, 71(4):893-9.

23. Keisuke S, Hiroshi Y, Kazuhiro A, Natsue K, Akito A, Kesatoki K, Masahiro Y: Sports Performance Benefits from Taking Natural Astaxanthin Characterized by Visual Acuity and Muscle Fatigue Improvement in Humans. J Clin Therap Med 2002, 18:1085-1100

24. Polotow TG, Vardaris CV, Mihaliuc AR, Goncalves MS, Pereira B, Ganini D, Barros MP: Astaxanthin supplementation delays physical exhaustion and prevents redox imbalances in plasma and soleus muscles of Wistar rats. Nutrients 2014, 6(12):581938.

25. Earnest CP, Lupo M, White KM, Church TS: Effect of astaxanthin on cycling time trial performance. Int J Sports Med 2011, 32(11):882-8.

26. Choi HD, Kim JH, Chang MJ, Kyu-Youn Y, Shin WG: Effects of astaxanthin on oxidative stress in overweight and obese adults. Phytother Res 2011, 25(12):1813-8.

27. Choi HD, Youn YK, Shin WG: Positive effects of astaxanthin on lipid profiles and oxidative stress in overweight subjects. Plant Foods Hum Nutr 2011, 66(4):363-9.

28. Karppi J, Rissanen TH, Nyyssonen K, Olsson AG, Voutilainen S, Salonen JT: Effects of astaxanthin supplementation on lipid peroxidation. Int J Vitam Nutr Res 2007, 77(1):3-11.

29. Jiang X, Zhu K, Xu Q, Wang G, Zhang J, Cao R, Ye J, et al.: The antidepressant-like effect of trans-astaxanthin involves the serotonergic system. Oncotarget 2017, 8(15):25552-25563.

30. Zhou XY, Zhang F, Hu XT, Chen J, Tang RX, Zheng KY, Song YJ: Depression can be prevented by astaxanthin through inhibition of hippocampal inflammation in diabetic mice. Brain Res 2017, 1657:262-268.

31. Jiang X, Chen L, Shen L, Chen Z, Xu L, Zhang J, Yu X: Trans-astaxanthin attenuates lipopolysaccharide-induced neuroinflammation and depressive-like behavior in mice. Brain Res 2016, 1649(Pt A):30-37.

32. Al-Amin MM, Sultana R, Sultana S, Rahman MM, Reza HM: Astaxanthin ameliorates prenatal LPS-exposed behavioral deficits and oxidative stress in adult offspring. BMC Neurosci 2016, 17:11.

33. Wibrand K, Berge K, Messaoudi M, Duffaud A, Panja D, Bramham CR, Burri L: Enhanced cognitive function and antidepressant-like effects after krill oil supplementation in rats. Lipids Health Dis 2013, 12:6.

34. Abadie-Guedes R, Guedes RC, Bezerra RS: The impairing effect of acute ethanol on spreading depression is antagonized by astaxanthin in rats of 2 young-adult ages. Alcohol Clin Exp Res 2012, 36(9):1563-7.

35. Mattei R, Polotow TG, Vardaris CV, Guerra BA, Leite JR, Otton R, Barros MP: Astaxanthin limits fish oil-related oxidative insult in the anterior forebrain of Wistar rats: putative anxiolytic effects? Pharmacol Biochem Behav 2011, 99(3):349-55.

36. Nishioka Y, Oyagi A, Tsuruma K, Shimazawa M, Ishibashi T, Hara H: The antianxietylike effect of astaxanthin extracted from Paracoccus carotinifaciens. Biofactors 2011, 37(1):25-30.

37. Liu X, Osawa T: Astaxanthin protects neuronal cells against oxidative damage and is a potent candidate for brain food. Forum Nutr 2009, 61:129-35. 
38. Curek GD, Cort A, Yucel G, Demir N, Ozturk S, Elpek GO, Savas B, et al.: Effect of astaxanthin on hepatocellular injury following ischemia/reperfusion. Toxicology 2010, 267(1-3):147-53.

39. Barros MP, Poppe SC, Bondan EF: Neuroprotective properties of the marine carotenoid astaxanthin and omega-3 fatty acids, and perspectives for the natural combination of both in krill oil. Nutrients 2014, 6(3):1293-317.

40. Dhillon VS, Fenech M: Mutations that affect mitochondrial functions and their association with neurodegenerative diseases. Mutat Res Rev Mutat Res 2014, 759:113.

41. Thayer JF, Lane RD: Claude Bernard and the heart-brain connection: further elaboration of a model of neurovisceral integration. Neurosci Biobehav Rev 2009, 33(2):81-8.

42. Mazzeo AT, Micalizzi A, Mascia L, Scicolone A, Siracusano L: Brain-heart crosstalk: the many faces of stress-related cardiomyopathy syndromes in anaesthesia and intensive care. Br J Anaesth 2014, 112(5):803-15.

43. Gunda S, Kanmanthareddy A, Atkins D, Bommana S, Pimentel R, Drisko J, Dibiase L, et al: Role of yoga as an adjunctive therapy in patients with neurocardiogenic syncope: a pilot study. J Interv Card Electrophysiol 2015, 43(2):105-10.

44. Shaffer F, McCraty R, Zerr CL: A healthy heart is not a metronome: an integrative review of the heart's anatomy and heart rate variability. Front Psychol 2014, 5:1040.

45. McCraty R, Atkinson M, Tomasino D: Impact of a workplace stress reduction program on blood pressure and emotional health in hypertensive employees. J Altern Complement Med 2003, 9(3):355-69.

46. McCraty R, Atkinson M, Tiller WA, Rein G, Watkins AD: The effects of emotions on short-term power spectrum analysis of heart rate variability. Am J Cardiol 1995, 76(14):1089-93. 